\title{
Translational potential of human embryonic and induced pluripotent stem cells for myocardial repair: Insights from experimental models
}

\author{
Chi-Wing Kong'; Fadi G. Akar2'; Ronald A. Li1,2 \\ ${ }^{1}$ Stem Cell \& Regenerative Medicine Program, Heart, Brain, Hormone \& Healthy Aging Research Center, and Department of Medicine, University of Hong Kong, Hong Kong, China; \\ ${ }^{2}$ Center of Cardiovascular Research, Mount Sinai School of Medicine, New York, New York, USA
}

\begin{abstract}
Summary
Heart diseases have been a major cause of death worldwide, including developed countries. Indeed, loss of non-regenerative, terminally differentiated cardiomyocytes (CMs) due to aging or diseases is irreversible. Current therapeutic regimes are palliative in nature, and in the case of end-stage heart failure, transplantation remains the last resort. However, this option is significantly hampered by a severe shortage of donor cells and organs. Human embryonic stem cells (hESCs) can self-renew while maintaining their pluripotency to differentiate into all cell types. More recently, direct reprogramming of adult somatic cells to become pluripotent hES-like cells (a.k.a. induced pluripotent stem cells or iPSCs) has been achieved. The availability of hESCs and iPSCs, and their successful differentiation into genuine human heart cells have enabled re-
\end{abstract}

searchers to gain novel insights into the early development of the human heart as well as to pursue the revolutionary paradigm of heart regeneration. Here we review our current knowledge of hESC-/iPSC-derived $\mathrm{CMs}$ in the context of two fundamental operating principles of CMs (i.e. electrophysiology and $\mathrm{Ca}^{2+}$-handling), the resultant limitations and potential solutions in relation to their translation into clinical (bioartificial pacemaker, myocardial repair) and other applications (e.g. as models for human heart disease and cardiotoxicity screening).

\section{Keywords}

Human embryonic stem cells, cardiomyocytes, induced pluripotent stem cells

\section{Correspondence to:}

Dr. Ronald Li

Stem Cell \& Regenerative Medicine Program

Heart, Brain, Hormone \& Healthy Aging Research Center,

and Department of Medicine

University of Hong Kong, Hong Kong, China

E-mail: ronaldli@hkucc.hku.hk or ronaldli@mssm.edu
Received: March 23, 2010

Accepted after minor revision: May 27, 2010

Prepublished online: June 10, 2010

doi:10.1160/TH10-03-0189

Thromb Haemost 2010; 104: 30-38

\section{Heart disease and motivation for cell-based therapy}

The heart beats with a regular rhythm to pump blood throughout the body. These pumping actions require the highly coordinated efforts of different types of cardiomyocytes (CMs) such as atrial, ventricular and pacemaker cells. These different CMs differ in their cellular morphologies, structural and functional properties. Autonomous rhythmic heart beats are modulated by sympathetic and parasympathetic means according to everyday needs. Normal rhythms originate in the sino-atrial (SA) node, a specialised cardiac tissue consisting of only a few thousands pacemaker cells. The SA node generates spontaneous rhythmic action potentials which subsequently propagate (i.e. the processes of pacing and conduction, respectively) to induce coordinated muscle contractions of the atria and ventricles for effective blood pumping. Since terminally-differentiated adult CMs lack the ability to regenerate, their malfunction or significant loss due to disease or aging can lead to lethal consequences such as heart failure and various lethal forms of arrhythmias. Heart transplantation for patients with end-stage heart failure is limited by the number of donor organs available; cell replacement therapy is a promising option for myocardial repair but limited by the availability of transplantable human CMs (e.g. human fetal CMs) due to practical and ethical reasons. As a result, transplantation of non-cardiac cells such as skeletal muscle myoblasts (SkM) and smooth muscle cells have been sought as potentially viable alternatives. However, the non-cardiac identity of these cell sources presented major limitations. For instance, the lack of electrical integration of SkM (conduction via gap junctions) after their autologous transplantation into the myocardium has been shown to underlie the generation of malignant ventricular arrhythmias, which led to the premature termination of clinical trials involving this specific cell source (1). Moreover, it is now well established that although bone marrow stem cells improve cardiac functions of ischaemic patients by promoting angiogenesis (2), they lack the capacity to transdifferentiate into cardiac muscle for myocardiogenesis (3), limiting their utility for cardiac repairs. Recently, adult cardiac resident stem cells have been explored as an autologous cell source. Here we will focus our discussion on human embryonic stem cells (hESC) and induced pluripotent stem cell (iPSC). 


\section{Human embryonic stem cells}

Upon fertilisation of an oocyte by sperm, the resultant zygote, which possesses the total potential (i.e. totipotency) to develop into all cell types including those necessary for embryonic development (such as extra-embryonic tissues), will undergo several rounds of cell division to become a compact ball of totipotent cells known as the morula. As the morula continues to grow ( $\sim 4$ days after fertilisation), its cells will migrate to form a more specialised hollow, fluid-filled structure known as the blastocyst consisting of an outer cell layer, the trophectoderm, and an inner cluster of cells collectively known as the inner cell mass (ICM). While the trophectoderm is committed to developing into extra-embryonic structures for supporting fetal development, the ICM will give rise to the embryo. The ICM retains the ability to form any cell of the body except the placental tissues (i.e. pluripotency). Embryonic stem cells (ESCs) are isolated from the ICM for cultivation in vitro. ESCs possess the ability to remain undifferentiated and propagate indefinitely in culture while maintaining their normal karyotypes and pluripotency to differentiate into the derivatives of all three embryonic germ layers (i.e. endoderm, mesoderm and ectoderm) and their lineage derivatives, including brain, blood, pancreatic, heart and other muscle cells. Pluripotent mammalian ESC lines were first derived from rodent blastocysts over 20 years ago, revolutionising mouse genetics and leading to the generation of the first transgenic animal. The first successful isolation of human ESCs (hESCs) by Thomson et al in 1998 further sparked tremendous international scientific interest: Since hESCs are immortal, they can potentially act as an unlimited exvivo source of even non-dividing, terminally differentiated cells for transplantation and cell-based human therapies. Indeed, hESCs can self-renew while maintaining their pluripotency to differentiate into all cell types (4), including CMs (5-9).

\section{Induced pluripotent stem cells}

Although hESC-derived CMs (hESC-CMs) have been reported to improve cardiac function in several animal models of myocardial infarction $(10,11)$, numerous hurdles need to be overcome before their clinical applications. For instance, generation of patient-specific cells for autologous transplantation has been pursued to avoid immune rejection of the transplanted grafts. This can be accomplished by somatic cell nuclear transfer (SCNT) of a patient's own nucleus to an enucleated donor oocyte to generate a cloned blastocyst for the derivation of patient-specific ESCs. To date, the establishment of non-human primate "patient-specific" ESCs (12) and cloning of several animal species $(13,14)$ have been successfully achieved via SCNT; however, significant ethical concerns remain for the application of SCNT in human therapies. Alternatively, direct reprogramming of adult somatic cells such as dermal fibroblasts to become pluripotent hES-like cells (a.k.a. induced pluripotent stem cells or iPSCs) has been recently reported, eliminating potential ethical concerns and making hES/iPS cell-based ther- apies one step closer to reality. Forced expression of four pluripotency genes (Oct3/4, Sox 2 and either c-Myc and Klf4 for mouse and human or Lin28 and Nanog for human) (15-18) suffices to reprogram mouse and human fibroblasts into iPSCs. iPSCs have morphology, proliferation, feeder dependence, surface markers, gene expression, epigenetic status, formation of embryoid bodies in vitro, promoter activities, telomerase activities, and in vivo teratoma formation similar to hESCs. Technically, iPSCs are cultured under conditions virtually identical to those for hESCs $(17,18)$. Using the same cardiac differentiation protocol originally developed for hESCs, iPSCs can be similarly differentiated into CMs (17).

\section{Directed cardiac differentiation}

Previous studies using CMs derived from murine (m) and human ESCs have provided significant insights into the development of mammalian hearts. In vitro ESC differentiation requires an initial step of forming three-dimensional aggregates called embryoid bodies (EBs), which in turn can differentiate into a wide variety of specialised cell types including CMs. Once differentiated, CMs can be readily identified even by visual inspection as spontaneouslycontracting outgrowths (9). The cardiogenicity of ESCs is heavily influenced by conditions such as the EB size, media composition (e.g. the presence of fetal bovine serum, inducing agents and other growth and transcription factors), the particular ESC lines being investigated, the time and duration of differentiation. The developmental changes of mESC-derived CMs correlates with the length of time in culture, mimicking that seen in myocardial development in vivo: primordial pacemaker-like and SA nodal pacemaker (early), atrial (intermediate) and ventricular (late) CMs appear and predominate at different stages during cardiac differentiation although a heterogenous population of all three CM types is almost always seen in mouse EBs. Terminal differentiation and derivation of specific CM types is an area of tremendous interests that requires further investigation. Morphologically, mESC-derived nodal cells are non-striated, small and round whereas the atrial and ventricular counterparts show organised myofibrillar and sarcomeric structures found in mature cardiac muscle cells. Some nodal CMs lack the contractile apparatus (e.g. leading pacemaker cells in the SA node centre), and thus do not beat (without coupling to muscle CMs) although they are electrically active; in contrast, mononucleated and rod-shaped striated atrial and ventricular derivatives are normally quiescent but are capable of beating upon stimulation by electrical signals from nodal or neighbouring CMs. Recently, several unique multipotent primordial cardiac progenitor populations have been identified (19-21). As an example, specification of human tripotent $\mathrm{KDR}^{\text {low }} / \mathrm{c}-\mathrm{kit}^{\text {neg }}$ cardiovascular progenitors (CP) into CMs has been reported (19). Stagespecific induction of hESCs with activin A, BMP4, bFGF, VEGF and DKK1 for driven mesodermal differentiation results in generation of a KDR ${ }^{\text {low }} / \mathrm{c}-\mathrm{kit}^{\text {neg }} \mathrm{CP}$ population which defines one of the earliest stages of human cardiac development. CP-containing "car- 


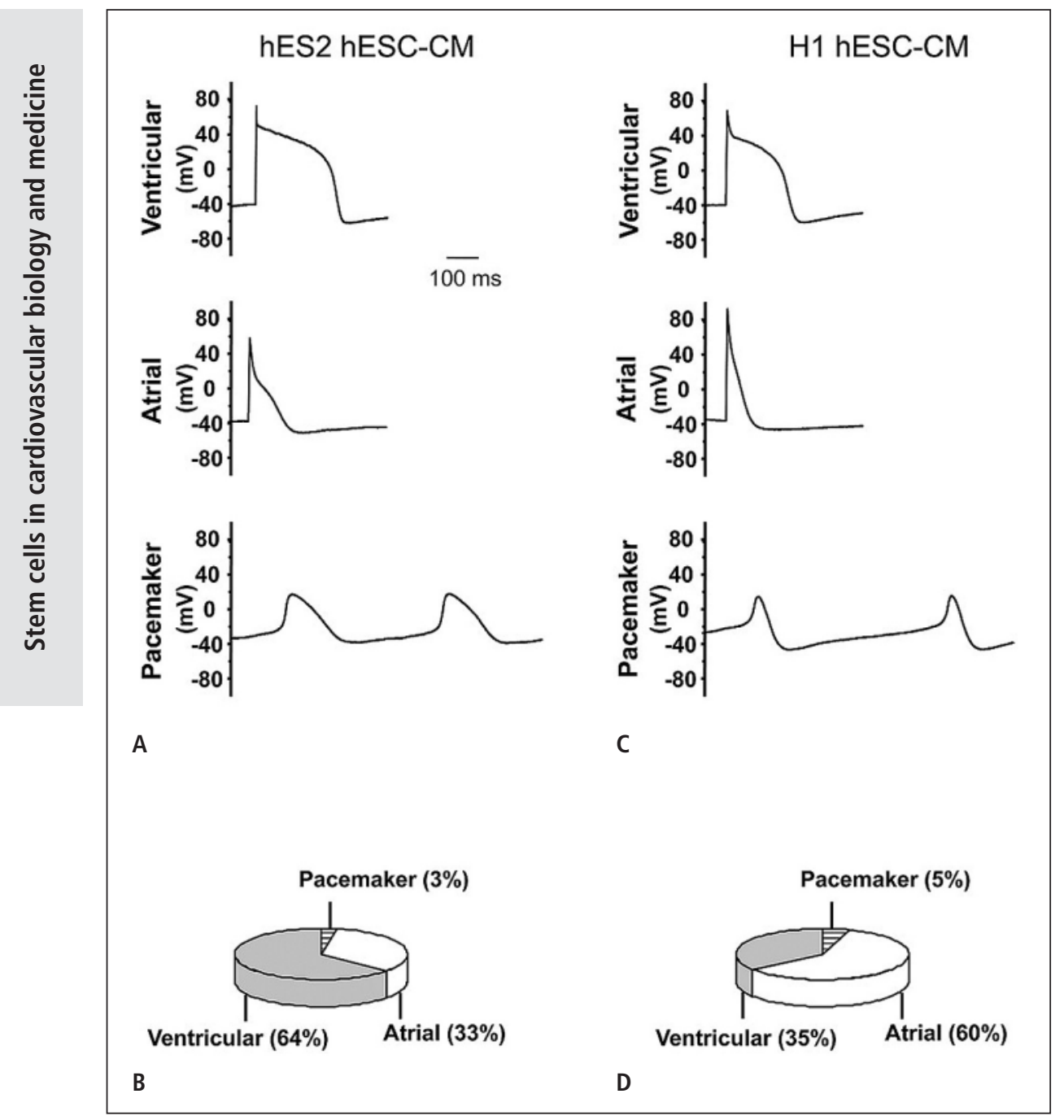

Figure 1: Action potentials $(A, C)$ and pie graphs (B, D) showing the \% distribution of chamber-specific CMs from HES2 and H1, respectively. Adapted from Moore et al. 2008. diospheres" derived from hESCs can efficiently differentiate into CMs in vitro (with $>50 \%$, as gauged by $\mathrm{CTNT}^{+}$cells). Further modifications of this protocol enable the induction of a large $\mathrm{CP}$ population, allowing the generation of highly enriched CMs even without the need for cell sorting. When properly instructed, CP can also differentiate into smooth muscle and endothelial cells.

\section{Functional and structural properties of hESC- and iPSC-derived cardiomyocytes}

The functional and structural properties of hESC-CMs have been investigated by us and several laboratories. Human ESC-CMs express cardiac-specific transcription factors and structural proteins (5-9). Like the murine counterpart, hESC-CMs derived from in vitro differentiation were highly heterogenous consisting of a combination of chamber-specific cell types. A directed differentiation protocol for deriving a particular chamber-specific type (e.g., the ventricular lineage), however, has not been established. Although all hESC lines are by definition pluripotent, we found that different lines have distinct cardiogenic potentials to become early ventricular-, atrial- and pacemaker-like derivatives as gauged by their signature action potential (AP) profiles. For instance, HES2 cells have a higher likelihood than $\mathrm{H} 1$ cells of differentiating into ventricular-like hESC-CMs (23) ( Fig. 1). In the sections that follow, we will focus our discussion on electrophysiology and $\mathrm{Ca}^{2+}$-handling, two fundamental operating properties of CMs.

\section{Functional but immature $\mathrm{Ca}^{2+}$-handling of hESC-CMs}

During an action potential (AP) of adult CMs, $\mathrm{Ca}^{2+}$ entry into the cytosol through sarcolemmal L-type $\mathrm{Ca}^{2+}\left(\mathrm{I}_{\mathrm{Ca}, \mathrm{L}}\right)$ channels triggers the release of $\mathrm{Ca}^{2+}$ from the intracellular $\mathrm{Ca}^{2+}$ stores (a.k.a. sarcoplasmic reticulum or SR) via the ryanodine receptors (RyR). This process, the so-called $\mathrm{Ca}^{2+}$-induced $\mathrm{Ca}^{2+}$-release (CICR) (24), escalates the cytosolic $\mathrm{Ca}^{2+}\left(\left[\mathrm{Ca}^{2+}\right]_{\mathrm{i}}\right)$ to activate the contractile apparatus for contrac- 
Figure 2: Functional but immature Ca2+-handling of hESC-CMs as compared to FLV-CMs and ALV-CMs. A) Representative tracings caffeine-induced $\mathrm{Ca}^{2+}$ transients of HES2-, H1-, and FLV-CMs. B) \% of caffeine-responsive and -insensitive cells. C) Expression of various $\mathrm{Ca}^{2+}$ handling proteins. $\beta$-actin was used as the loading control. D) Immunostaining of RyRs. Adapted from Liu et al. 2007.

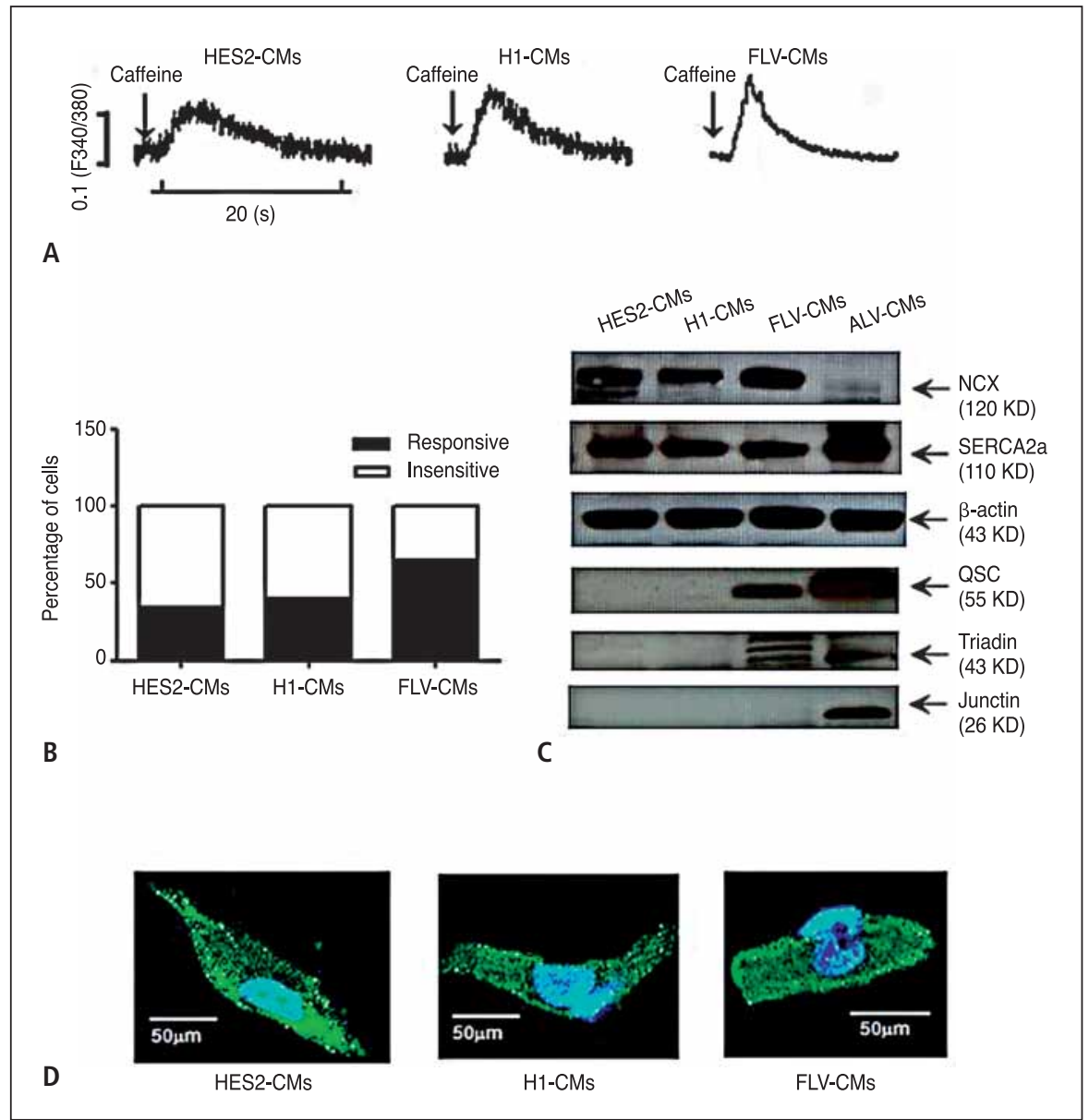

tion. For relaxation, elevated $\left[\mathrm{Ca}^{2+}\right]_{i}$ gets pumped back into the SR by the sarco/endoplasmic reticulum $\mathrm{Ca}^{2+}$-ATPase (SERCA) and extruded by the $\mathrm{Na}^{+}-\mathrm{Ca}^{2+}$ exchanger (NCX) to return to the resting $\left[\mathrm{Ca}^{2+}\right]_{\mathrm{i}}$ level. Such a rise and subsequent decay of $\left[\mathrm{Ca}^{2+}\right]_{\mathrm{i}}$ is known as $\mathrm{Ca}^{2+}$ transient. Both the contractile force (inotropic) and frequency (chronotropic) of CMs are dependent on the amplitude and kinetic properties of $\mathrm{Ca}^{2+}$ transients. In mature ventricular CMs, CICR is optimised by the presence of t-tubules, invaginations in the sarcolemmal membrane that concentrates $\mathrm{I}_{\mathrm{Ca}, \mathrm{L}}$ channels and brings them spatially closer to RyRs $(25,26)$. With a minimised $\mathrm{Ca}^{2+}$ diffusion distance between $\mathrm{I}_{\mathrm{Ca}, \mathrm{L}}$ and RyRs, SR deep in ventricular CMs with large cross-sectional area can participate in CICR without significant time lags. This increased efficiency is demonstrated by a uniform increase in cytosolic $\mathrm{Ca}^{2+}$ across the transverse section of the cell (with simultaneous recruitment of all SRs). Such a uniform $\mathrm{Ca}^{2+}$ wave starkly contrasts the U-shaped $\mathrm{Ca}^{2+}$ wave propagation from the periphery to the center in a de-tubulated ventricular or atrial CM (that lacks t-tubules) (25). The U-shaped waves result from a time delay that is proportional to the diffusion distance squared in recruiting the $\mathrm{Ca}^{2+}$ stores at the cell centre (27).

Given the central importance of CICR in cardiac excitationcontraction (EC) coupling, proper $\mathrm{Ca}^{2+}$ handling properties of hESC/iPSC-CMs are therefore crucial for their successful functional integration with the recipient heart after transplantation. In- deed, abnormal $\mathrm{Ca}^{2+}$ handling, as in the case of heart failure, can even be arrhythmogenic (e.g. by causing delayed afterdepolarization) (24). In murine (m) ESC-CMs, both the SR load and RyR are essential for regulating contractions even at very early developmental stages $(28,29)$. As for the human counterpart, in 2006 Dolnikov et al. were the first to study the $\mathrm{Ca}^{2+}$-handling properties of hESC-CMs in detail (30). They reported that $\mathrm{Ca}^{2+}$ transients recorded from spontaneously beating or electrically stimulated hESC-CMs respond to neither caffeine nor ryanodine; hESC-CMs recorded as beating clusters also displayed a negative force-frequency relationship that is different from adult CMs. Based on these observations, the authors concluded that hESC-CMs are immature and do not express functional SRs, and that their contractions result from trans-sarcolemmal $\mathrm{Ca}^{2+}$ influx (rather than $\mathrm{Ca}^{2+}$ release from the SR). Due to the paucity of further hESC-CM data, we sought to help define the poorly known $\mathrm{Ca}^{2+}$-handling properties by recording and comparing $\mathrm{Ca}^{2+}$ transients from hESC-CMs and human fetal left ventricular (LV) CMs (16-18 weeks) ( Fig. 2A). In stark contrast to the previous study, we demonstrated the presence of functional SRs even in hESC-CMs that were younger (31) (18-24 vs. 55-day old post-differentiation of Dolnikov et al.). Upon electrical stimulation, hESC- and fetal LV-CMs generated similar $\mathrm{Ca}^{2+}$ transients. However, caffeine induced $\mathrm{Ca}^{2+}$ release in $65 \%$ of fetal LV-CMs and only $40 \%$ of $\mathrm{H1}-$ and HES2-CMs. Rya- 


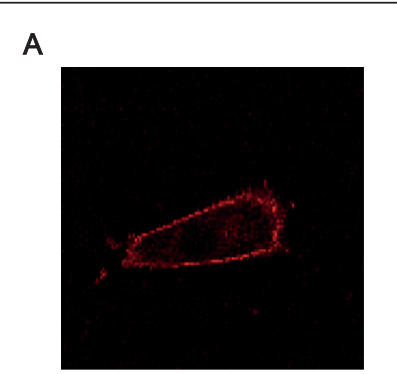

C

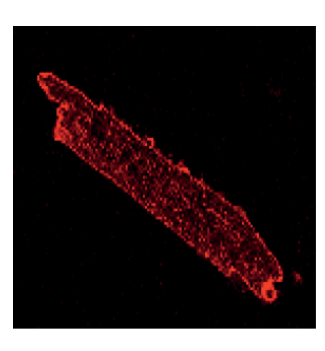

B

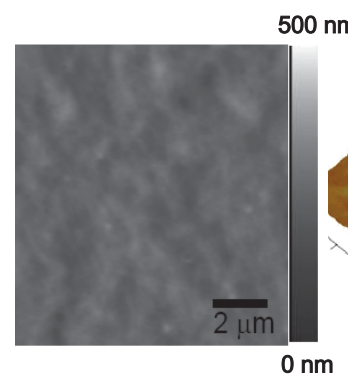

D

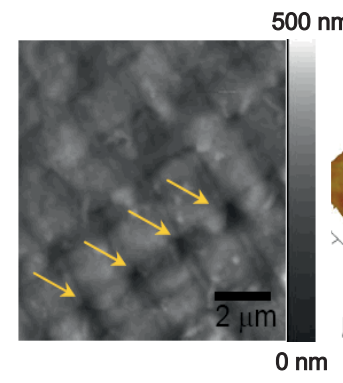

$00 \mathrm{~nm}$

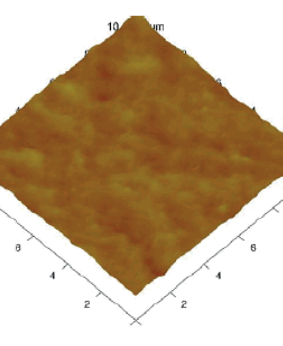

$500 \mathrm{~nm}$

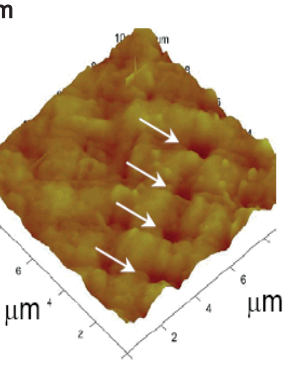

E

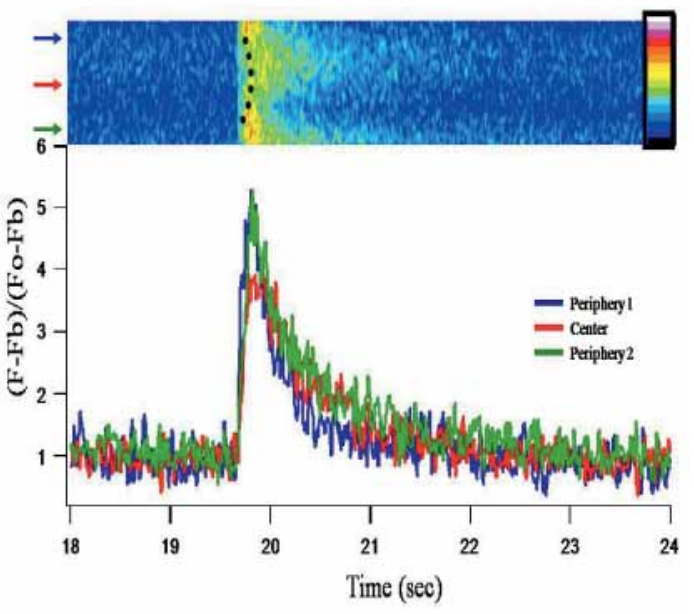

Figure 3: T-tubule imaging of a hESC-CM and a mature ventricular CM. Di-8-ANEPPS confocal microscopic images of a hESC-CM (A) did not show intracellular fluorescent spots like those in an adult guinea pig ventricular CM (C) suggesting the absence of $t$-tubules. The absence of $t$-tubules in ESC-CMs was further confirmed by atomic force microscopy (AFM) imaging of an adult ventricular cardiomyocyte (D) showing regularly spaced pores in the sarcolemma that coincide with the Z-lines, while hESC-CM (B) surface showed comparatively smoother topology with no presence of invaginations that are indicative of $\mathrm{t}$-tubules. E) Electrically induced $\mathrm{Ca}^{2+}$ transient in hESC-CMs. Top: Time progression linescans of pseudo-colored transient increase in intracellular $\mathrm{Ca}^{2+}$ across the mid-plane of a hESC-CM showed a U-shaped wavefront. Bottom: Quantified $\mathrm{Ca}^{2+}$ transient of linescans of the top panel. Adapted from Lieu et al. 2009. nodine significantly reduced the electrically-evoked $\mathrm{Ca}^{2+}$ transient amplitudes and slowed the upstroke of caffeine-responsive HES2and H1-CMs but NOT caffeine-insensitive cells; thapsigargin, a SERCA inhibitor, similarly reduced the amplitude and slowed the decay of only caffeine-responsive HES2- and H1-CMs (31). Thus, the discrepancy can be largely attributed to the newly identified caffeine-responsive population. NCX protein expression is highest in hESC-CMs, but intermediate and weakest in fetal and adult LV$\mathrm{CMs}$, respectively. We later confirmed that the same trend is also seen at the functional level (32). Although highest in adult LVCMs, SERCA2a is already substantially and comparably expressed in H1-, HES2-, and fetal LV-CMs. RyR is expressed in hESC-CMs and fetal LV-CMs, but the organised pattern for adult LV-CMs is not observed (due to the lack of t-tubules in hESC-CMs). On the far end, the regulatory proteins junctin, triadin, and calsequestrin (CSQ) are expressed in adult LV-CMs but completely absent in hESC-CMs. Furthermore, hESC-CMs do not display t-tubules that are seen in normal adult human atrial and ventricular CMs (33) ( Fig. 3). Consistent with the lack of t-tubules, the $\mathrm{Ca}^{2+}$ wavefront of hESC-CMs is U-shaped, suggesting inefficient CICR or time delay in activation of RyRs at the centre with faster and greater magnitude of $\mathrm{Ca}^{2+}$ transient increase at the periphery relative to the central part of the cells (33). Table 1 summarises some $\mathrm{Ca}^{2+}$-handling properties of hESC-, fetal and adult CMs.

$\mathrm{Ca}^{2+}$ homeostasis is dependent on such $\mathrm{Ca}^{2+}$-handling proteins as $\mathrm{I}_{\mathrm{Ca}, \mathrm{L}}$ channels, RyR, SERCA and NCX. RyRs are arranged in large organised arrays (up to $200 \mathrm{~nm}$ in diameter with more than 100 RyRs) at the junctions between the SR and sarcolemma (i.e. t-tubules) beneath $\mathrm{I}_{\mathrm{Ca}, \mathrm{L}}$ channels (34). These arrays constitute a large functional $\mathrm{Ca}^{2+}$ release complex. RyRs are also coupled to other proteins at the luminal SR surface such as triadin, junctin and calsequestrin (CSQ) (35). As the most abundant, high-capacity but low-infinity Ca-binding protein in the SR, the cardiac isoform CSQ2 can store up to $20 \mathrm{mM} \mathrm{Ca}^{2+}$ while buffering the free SR $\left[\mathrm{Ca}^{2+}\right]$ at $\sim 1 \mathrm{mM}$ (36). This allows repetitive muscle contractions without run-down. CSQ2 also coordinates the rates of $\mathrm{SR} \mathrm{Ca}^{2+}$ release and loading by modulating RyR activities. Indeed, the SR $\mathrm{Ca}^{2+}$ content affects the amount of $\mathrm{Ca}^{2+}$ released via $\mathrm{CICR}(37,38)$. For a given $\mathrm{I}_{\mathrm{Ca}, \mathrm{L}}$ trigger, a high SR $\mathrm{Ca}^{2+}$ load enhances the open probability of RyRs while directly providing more $\mathrm{Ca}^{2+}$ available for release (39). By contrast, $\mathrm{I}_{\mathrm{Ca}}$ can no longer cause CICR when the $\mathrm{SR} \mathrm{Ca}^{2+}$ content is sufficiently low. Mechanistically, CSQ senses the levels of luminal $\mathrm{Ca}^{2+}$ and effects RyRs via triadin and junctin. For instance, when SR $\mathrm{Ca}^{2+}$ declines (e.g. during $\mathrm{Ca}^{2+}$ release), the increased level of $\mathrm{Ca}^{2+}$-free CSQ deactivates RyRs by binding via triadin and junction; alternatively, SR $\mathrm{Ca}^{2+}$ reload (e.g. upon relaxation when CICR terminates) relieves the CSQ2-mediated inhibition of RyRs $(36,40)$. Thus, CSQ2 is an important determinant of the SR load. Interestingly, CSQ can activate purified RyRs in the absence of triadin and junction $(41,42)$. As mentioned, CSQ is completely absent in hESC-CMs. We hypothesised that gene transfer of CSQ in hESC-CMs suffices to induce functional im- 
Table 1: Summary of differences. H1- and HES2-CMs have comparable trends and are collectively referred to as hESC-CMs.

\begin{tabular}{|c|c|c|c|c|}
\hline & & hESC-CMs & Fetal LVCMs & Adult LVCMs \\
\hline \multirow{6}{*}{$\begin{array}{l}\text { Expression levels of } \\
\mathrm{Ca}^{2+} \text {-handling proteins }\end{array}$} & RyR & ++ & ++ & ++++ \\
\hline & SERCA & +++ & +++ & ++++ \\
\hline & Phospholamban & - & ++ & ++++ \\
\hline & $\mathrm{CSQ} / \mathrm{Tdn} / \mathrm{Jtn}$ & - & + & ++++ \\
\hline & Calreticulin & ++++ & ++++ & + \\
\hline & NCX & +++ & ++++ & + \\
\hline \multirow{4}{*}{$\begin{array}{l}\mathrm{Ca}^{2+} \text { transient } \\
\text { properties }\end{array}$} & Basal $\left[\mathrm{Ca}^{2+}\right]_{\mathrm{i}}$ & ++ & +++ & ++++ \\
\hline & Amplitude & ++ & ++ & ++++ \\
\hline & Decay & ++ & ++ & ++++ \\
\hline & Upstroke & ++ & ++ & ++++ \\
\hline
\end{tabular}

provement of SR. Figure 4 shows that transduction of hESCCMs by the adenovirus Ad-CMV-CSQ-IRES-GFP (Ad-CSQ) significantly increased the transient amplitude, upstroke velocity and decay in comparison to the Ad-CMV-GFP and Ad-CMV-CSQ $\Delta$ IRES-GFP control groups. CSQ $\Delta$ is a non-functional version of CSQ with 53-869bp of the coding sequence deleted (43). Ad-CSQ also increased the $\mathrm{SR} \mathrm{Ca}^{2+}$ content (as tested by caffeine), without altering $\mathrm{I}_{\mathrm{Ca}} \mathrm{L}$, suggesting that the improved transient was not simply due to more $\mathrm{Ca}^{2+}$ influx for CICR.

\section{Immature electrophyshiological properties of hESC-CMs}

Adult LV CMs are normally electrically silent-yet-excitable upon stimulation. To assess the electrical maturity of ventricular hESC$\mathrm{CMs}$, we investigated their ability to generate APs. The majority of ventricular hESC-CMs were spontaneously firing, exhibiting a high degree of automaticity. The remaining quiescent cells could elicit single APs upon stimulation, indicating that their excitability was intact. However, these silent-yet-excitable ventricular hESCCMs displayed a prominent "phase 4-like" depolarisation, a known substrate for delayed after depolarisation (DAD). Furthermore, their resting membrane potentials (RMPs) were significantly depolarised. Collectively, these immature electrical properties of hESC-CMs are signatures of arrhythmogenic, failing adult ventricular CMs. Thus, strategies for facilitated maturation need to be developed. Building upon our series of cardiac automaticity studies (44-48), we first identified the lack of Kir2.1-encoded $\mathrm{I}_{\mathrm{K} 1}$ in hESC-CMs as the primary mechanistic contributor to the immature proarrhythmic electrophysiological properties. Forced Kir2.1 expression alone sufficed to render the electrical phenotype indistinguishable from that of primary adult ventricular cells. Based on these proof-of-concept data that the developmentally arrested $\mathrm{Ca}^{2+}$ and electrophysiological phenotypes of hESC-CMs can be rescued, we are currently developing a non-genetic, non-pharmacologic method for reproducibly driving global maturation, by targeting the micro-environmental niches and other non-cell autonomous means.

\section{Human iPSC-derived cardiomyocytes}

We have previously reported that both human ESCs and iPSCs express several specialised ion channel proteins that appear to regulate cell proliferation and cell cycle $(49,50)$. Using the same methods for hESCs, human iPSCs can likewise differentiate into CMs with cardiac-specific molecular, structural, electrophysiological and $\mathrm{Ca}^{2+}$-handling properties (51). The iPSC-derived CMs expressed cardiac-specific transcription factors and structural proteins (22). Functional CMs with nodal-, atrial-, or ventricular-like

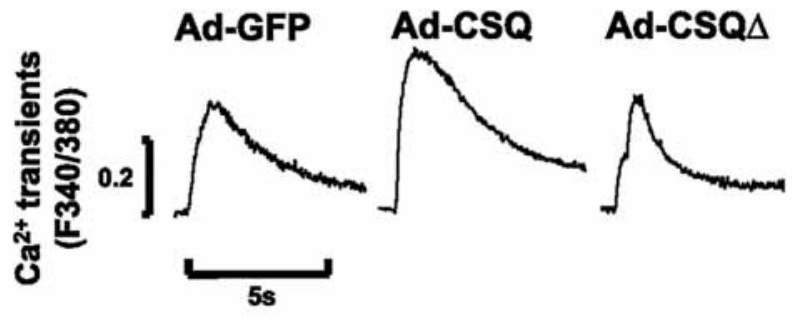

A

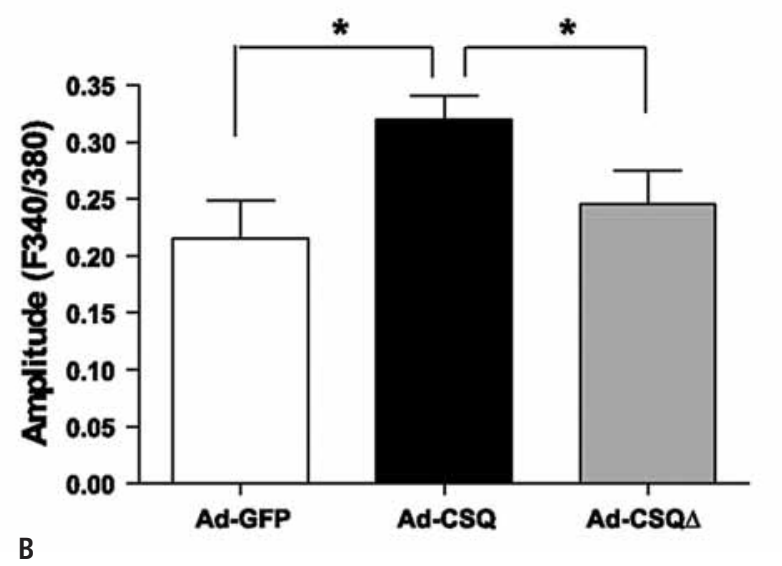

Figure 4: Effect of CSQ overexpression on hESC-CMs. A) Representative electrically-induced $\mathrm{Ca}^{2+}$ transient tracings for Ad-GFP $(n=12)$ and Ad-CSQ $(n=29)$ transduced hESC-CMs. B) Bar graphs of amplitude. ${ }^{*} P<0.05$, ${ }^{* *} P<0.01$. Adapted from Liu et al. 2009. 
electrophysiological phenotypes have also been observed (51). Multielectrode array recordings established the development of a functional syncytium with stable pacemaker activity and action potential propagation. Positive and negative chronotropic responses were induced by application of isoproterenol and carbamylcholine, respectively (22). Like hESC-CMs, iPSC-CMs also display immature $\mathrm{Ca}^{2+}$-handling properties with smaller transient amplitude and slowed kinetics (unpublished). Depending on the method and conditions of reprogramming, however, some iPSCCMs appear to be more resistant to maturation methods that work effectively for hESC-CMs. In addition to clinical applications, the ability to generate patient-specific hiPSC-CMs also provides an opportunity to develop novel in vitro models of cardiac disorders. Indeed, these in vitro models could serve as a platform for investigating arrhythmia mechanisms and for predicting efficacy of pharmacological therapies.

\section{Electronic pacemakers for heart rhythm disorders}

Normal rhythms originate in the sino-atrial node (SAN), a specialised cardiac tissue consisting of only a few thousands pacemaker cells $(52,53)$. Malfunction of cardiac pacemaker cells leads to rhythm generation disorders. Traditional treatments require pharmacological intervention and/or implantation of electronic pacemakers. While such therapy is effective, it is also associated with significant risks (e.g. infection, haemorrhage, lung collapse and death) and expenses ( $>600,000$ cardiac pacemakers are implanted annually worldwide). Other disadvantages include limited battery life (replaced every 5-10 years), permanent implantation of catheters, and lack of autonomic neurohumoral responses. As for paediatric patients with conditions such as symptomatic bradycardias, bradycardia-dependent ventricular arrhythmias and other postoperative arrhythmias that require electronic pacemakers for life-sustaining rhythms, several considerations can complicate their management (54-58). For instance, somatic growth may result in lead tension and thereby increase the risk of lead dislodging and fracturing that are fairly common in active young patients. The placement of an electronic system could also be hindered by congenital heart defects and structural abnormalities.

Unlike rhythmically AP-firing pacemaker cells, adult atrial and ventricular muscle cells are normally electrically silent unless they get stimulated by signals transmitted from neighbouring cells that originate from the SAN. This quiescent nature of cardiac muscle CMs is due to the absence of $\mathrm{I}_{\mathrm{f}}$ and the intense expression of $\mathrm{I}_{\mathrm{K} 1}$ (a.k.a. the inward-rectifier $\mathrm{K}^{+}$current), encoded by the Kir2 gene family, which stabilises a negative resting membrane potential (RMP -80 mV). Given the limitations of electronic pacemakers, several gene- and cell-based approaches have been explored to confer upon normally-quiescent cardiac muscle cells the ability to intrinsically fire APs similar to genuine nodal pacemaker cells as potential biological alternatives or supplements to devices. In addition to the translational potential, these studies also shed mech- anistic insights into cardiac automaticity. Miake et al. demonstrated that genetic suppression of $\mathrm{I}_{\mathrm{K} 1}$ in normally-silent ventricular myocytes by $>80 \%$ can cause spontaneous firing activity in a binary "on-and-off" fashion (59). The induced frequency is only a third of normal, and as such not suitable for acting as a reliable biological pacemaker. Alternatively, we $(44,48,60)$ and others (61) have chosen to employ a genuine pacemaker gene product - the $\mathrm{HCN}$ channels. Since the four HCN isoforms co-assemble to form heteromultimeric channels, the molecular identity of endogenous $\mathrm{I}_{\mathrm{f}}$ is complex and therefore difficult to reproduce via genetic expression of a single HCN isoform. Furthermore, subunit interactions of HCN are poorly defined. Indeed, overexpression of WT HCN1 (48) or HCN2 (62) channels alone in quiescent ventricular $\mathrm{CMs}$ is insufficient to cause oscillations. We overcame this hurdle by protein engineering of $\mathrm{HCN}$ to create a single construct that mimics native nodal $I_{f}(63-65)$, thereby enabling us to subsequently construct a functional bioartificial SAN (bio-SAN) in a large animal (swine) model of sick sinus syndrome (SSS) $(48,60)$. Side-by-side comparison in this SSS porcine model shows that bioSAN significantly reduces the dependence on device-supported pacing by electronic pacemaker from $\sim 85 \%$ to $\sim 15 \%$ in the same animal after receiving our treatment.

Alternatively, pluripotent human embryonic stem cells (hESCs) can be differentiated into pacemaker-like derivatives for transplantation to recreate an in vivo pacemaker $(8,66)$. To create biological pacemakers, human mesenchymal stem cells (hMSCs) pre-transfected with HCN2 channels have also been employed as a delivery vehicle for introducing $\mathrm{I}_{\mathrm{f}}$ into neighboring $\mathrm{CMs}$ via gap junctionmediated coupling $(67,68)$. The stability of such coupling between non-cardiac cells and the myocardium needs to be considered and requires further investigations. Along the same line, ventricular myocytes have been converted into pacemaker-like heterokaryons via chemically-induced fusion with fibroblasts transduced to express HCN1 channels (69). When injected into the guinea pig left ventricle (LV), electrocardiography (ECG) confirmed ectopic rhythms. Previous studies have indicated that similar heterokaryons can remain stable for several months (70-72).

\section{Myocardial repair}

Myocardial infarction remains the major worldwide cardiovascular disorder in humans. Immediately after a heart attack, oxygen starvation of myocardial tissues leads to cell death, and in the absence of immediate thrombolytic treatment of the blocked coronary artery, the damage is often irreversible and the heart is permanently impaired. Despite significant improvements in the management of most cardiovascular disorders, sudden cardiac death (SCD) due to ventricular arrhythmias remains a leading cause of morbidity and mortality in the industrialised world, claiming well over 300,000 lives annually in the United States alone. Current treatment options for arrhythmias in patients with heart failure have been especially disappointing, in many cases promoting rather than preventing SCD. Longer term, the remaining CMs hy- 
pertrophy in an attempt to meet the functional demands, while cardiac fibroblasts secrete collagen and other extracellular matrix proteins during scar formation, which may further impair ventricular function. Indeed, myocardial tissue recovery is particularly limited by its intrinsic inability to regenerate. Palliative interventions to treat damaged heart muscle include changes in life-style, medications that reduce functional requirements or limit certain aspects of remodelling, or surgical interventions that improve cardiac function. In severe cases, transplantation is the only option, and today's most urgent problem in transplantation is the lack of suitable donor organs and tissues. As mentioned, damaged myocardium could be treated by cell transplantation/replacement therapy. The lack of effective and safe therapeutic options for heart failure patients, coupled with a growing understanding of cardiac regenerative processes and stem cell biology have led to the development of various cell-based therapies that have shown significant promises in at least partially restoring cardiac function by reducing infarct size. Potential and actual sources of cells or tissues are self (autologous), same species (allogeneic), different species (xenographic), primary or immortalised cell lines, and embryonic or adult stem cell-derived donor cells.

Pathophysiological remodeling of cardiac function occurs at multiple levels, spanning the spectrum from molecular and subcellular changes to those occurring at the organ-system levels. Complex alterations in a host of ion channels, $\mathrm{Ca}^{2+}$-cycling proteins, and gap junction-related molecules modulates key electrophysiological properties, predisposing to arrhythmias caused by enhanced automaticity, triggered activity, and reentry. Heart failure-induced ion channel dysfunction prolongs the AP, increases spatiotemporal gradients of repolarisation, promotes the formation of arrhythmogenic triggers and results in conduction abnormalities. Cell-based therapies can significantly improve LV function. As such, these therapies may activate complex signalling processes that reverse-remodel the failing heart, and therefore prevent the incidence of arrhythmias. On the other hand, cell-based therapies might also alter the RMP, produce abnormal triggers, promote electrical heterogeneities, modulate conduction, and favour reentrant excitation. As already described, of major concern is the fact that hESC- and iPSC-CMs display a range of functional and structural properties that are remarkably similar to those of immature or failing heart cells. These properties along with a detailed investigation of their electrophysiological consequences require careful investigation before their clinical efficacy can be further assessed.

\section{Conclusion}

The availability of hESC and iPSC and their successful differentiation into genuine human heart cells have enabled clinicians and scientists to gain insights into the early development of the human heart as well as to pursue to the revolutionary paradigm of heart regeneration. However, hESC- and iPSC-derived CMs appear to be both structurally and functionally immature. One of the most im- portant factors to consider before any potential benefits of hESCCMs are clinically assessed would be to ensure their safety. Furthermore, the successful use of derived CMs as human heart disease models and cardiotoxicity screening tools depends on their ability to recapitulate the properties of their adult counterparts. Further studies are required to promote their maturation. When combined with other advances in driven differentiation and cardiovascular progenitor identification (19-21), the approaches can facilitate the translation of hESC/iPSCs into clinical and other applications.

\section{Acknowledgement}

This work was supported by grants from the NIH - R01 HL72857 (to R.A.L.), the CC Wong Stem Cell Foundation Fund (to R.A.L.) and the University Development Fund (to C-W.K. and R.A.L.).

\section{References}

1. Abraham MR, et al. Antiarrhythmic engineering of skeletal myoblasts for cardiac transplantation. Circulation Res 2005; 97: 159-167.

2. Tse HF, et al. Angiogenesis in ischaemic myocardium by intramyocardial autologous bone marrow mononuclear cell implantation. Lancet 2003; 361: 47-49.

3. Murry CE, et al. Haematopoietic stem cells do not transdifferentiate into cardiac myocytes in myocardial infarcts. Nature 2004; 428: 664-668.

4. Thomson JA, et al. Embryonic stem cell lines derived from human blastocysts. Science 1998; 282: 1145-1147.

5. He JQ, et al. Human embryonic stem cells develop into multiple types of cardiac myocytes: Action potential characterization. Circulation Res 2003; 93: 32-39.

6. Mummery C, et al. Differentiation of human embryonic stem cells to cardiomyocytes: Role of coculture with visceral endoderm-like cells. Circulation 2003; 107: $2733-2740$.

7. $\mathrm{Xu} \mathrm{C}$, et al. Characterization and enrichment of cardiomyocytes derived from human embryonic stem cells. Circulation Res 2002; 91: 501-508.

8. Xue $\mathrm{T}$, et al. Functional integration of electrically active cardiac derivatives from genetically engineered human embryonic stem cells with quiescent recipient ventricular cardiomyocytes: Insights into the development of cell-based pacemakers. Circulation 2005; 111: 11-20.

9. Kehat I, et al. Human embryonic stem cells can differentiate into myocytes with structural and functional properties of cardiomyocytes. J Clin Invest 2001; 108: 407-414.

10. Laflamme MA, et al. Cardiomyocytes derived from human embryonic stem cells in pro-survival factors enhance function of infarcted rat hearts. Nat Biotechnol 2007; 25: 1015-1024.

11. Caspi O, et al. Transplantation of human embryonic stem cell-derived cardiomyocytes improves myocardial performance in infarcted rat hearts. J Am Coll Cardiol 2007; 50: 1884-1893.

12. Byrne JA, et al. Producing primate embryonic stem cells by somatic cell nuclear transfer. Nature 2007; 450: 497-502.

13. Yang X, et al. Nuclear reprogramming of cloned embryos and its implications for therapeutic cloning. Nat Genet 2007; 39: 295-302.

14. Kishigami S, et al. Production of cloned mice by somatic cell nuclear transfer. Nat Protoc 2006; 1: 125-138.

15. Takahashi K, Yamanaka S. Induction of pluripotent stem cells from mouse embryonic and adult fibroblast cultures by defined factors. Cell 2006; 126: 663-676.

16. Meissner A, et al. Direct reprogramming of genetically unmodified fibroblasts into pluripotent stem cells. Nat Biotechnol 2007; 25: 1177-1181.

17. Takahashi $\mathrm{K}$, et al. Induction of pluripotent stem cells from adult human fibroblasts by defined factors. Cell 2007; 131: 861-872.

18. Yu J, et al. Induced pluripotent stem cell lines derived from human somatic cells. Science 2007; 318: 1917-1920.

19. Yang L, et al. Human cardiovascular progenitor cells develop from a kdr+ embryonic-stem-cell-derived population. Nature 2008; 453: 524-528. 
20. Bu L, et al. Human isll heart progenitors generate diverse multipotent cardiovascular cell lineages. Nature 2009; 460: 113-117.

21. Moretti A, et al. Multipotent embryonic isl1+ progenitor cells lead to cardiac, smooth muscle, and endothelial cell diversification. Cell 2006; 127: 1151-1165.

22. Zwi L, et al. Cardiomyocyte differentiation of human induced pluripotent stem cells. Circulation 2009; 120: 1513-1523.

23. Moore JC, et al. Distinct cardiogenic preferences of two human embryonic stem cell (hesc) lines are imprinted in their proteomes in the pluripotent state. Biochem Biophys Res Commun 2008; 372: 553-558.

24. Bers DM. Cardiac excitation-contraction coupling. Nature 2002; 415: 198-205.

25. Brette F, Orchard C. T-tubule function in mammalian cardiac myocytes. Circulation Res 2003; 92: 1182-1192.

26. Brette F, Orchard C. Resurgence of cardiac t-tubule research. Physiology 2007; 22: 167-173.

27. Song LS, et al. Calcium biology of the transverse tubules in heart. Ann NY Acad Sci 2005; 1047: 99-111.

28. Fu JD, et al. Crucial role of the sarcoplasmic reticulum in the developmental regulation of $\mathrm{Ca}^{2+}$ transients and contraction in cardiomyocytes derived from embryonic stem cells. Faseb J 2006; 20: 181-183.

29. Fu JD, et al. Developmental regulation of intracellular calcium transients during cardiomyocyte differentiation of mouse embryonic stem cells. Acta Pharmacol Sin 2006; 27: 901-910.

30. Dolnikov K, et al. Functional properties of human embryonic stem cell-derived cardiomyocytes: Intracellular ca2+ handling and the role of sarcoplasmic reticulum in the contraction. Stem Cells 2006; 24: 236-245.

31. Liu J, et al. Functional sarcoplasmic reticulum for calcium-handling of human embryonic stem cell-derived cardiomyocytes: Insights for driven maturation. Stem Cells 2007; 25: 3038-3044.

32. Fu JD, et al. $\mathrm{Na}^{+} / \mathrm{Ca}^{2+}$ exchanger is a determinant of excitation-contraction coupling in human embryonic stem cell-derived ventricular cardiomyocytes. Stem Cells Dev 2009; epub ahead of print.

33. Lieu DK, et al. Absence of transverse tubules contributes to non-uniform $\mathrm{Ca}^{2+}$ wavefronts in mouse and human embryonic stem cell-derived cardiomyocytes. Stem Cells Dev 2009; 18: 1493-1500.

34. Franzini-Armstrong $\mathrm{C}$, et al. Shape, size, and distribution of $\mathrm{Ca}(2+)$ release units and couplons in skeletal and cardiac muscles. Biophys J 1999; 77: 1528-1539.

35. Zhang L, et al. Complex formation between junctin, triadin, calsequestrin, and the ryanodine receptor. Proteins of the cardiac junctional sarcoplasmic reticulum membrane. J Biol Chem 1997; 272: 23389-23397.

36. Beard NA, et al. Calsequestrin and the calcium release channel of skeletal and cardiac muscle. Progr Biophys Mol Biol 2004; 85: 33-69.

37. Bassani JW, et al. Fractional SR Ca release is regulated by trigger Ca and SR Ca content in cardiac myocytes. Am J Physiol 1995; 268: C1313-C1319.

38. Shannon TR, et al. Potentiation of fractional sarcoplasmic reticulum calcium release by total and free intra-sarcoplasmic reticulum calcium concentration. Biophys J 2000; 78: 334-343.

39. Lukyanenko $V$, et al. Regulation of calcium release by calcium inside the sarcoplasmic reticulum in ventricular myocytes. Pflugers Arch 1996; 432: 1047-1054.

40. Gyorke I, et al. The role of calsequestrin, triadin, and junctin in conferring cardiac ryanodine receptor responsiveness to luminal calcium. Biophys J 2004; 86: 2121-2128.

41. Szegedi C, et al. Calsequestrin: More than 'only' a luminal ca2+ buffer inside the sarcoplasmic reticulum. Biochem J 1999; 337: 19-22.

42. Ohkura $\mathrm{M}$, et al. Dual regulation of the skeletal muscle ryanodine receptor by triadin and calsequestrin. Biochemistry 1998; 37: 12987-12993.

43. Terentyev D, et al. Calsequestrin determines the functional size and stability of cardiac intracellular calcium stores: Mechanism for hereditary arrhythmia. Proc Natl Acad Sci USA 2003; 100: 11759-11764.

44. Azene EM, et al. Non-equilibrium behavior of hon channels: Insights into the role of hon channels in native and engineered pacemakers. Cardiovasc Res 2005; 67: 263-273.

45. Lieu DK, et al. Overexpression of hcn-encoded pacemaker current silences bioartificial pacemakers. Heart Rhythm 2008; 5: 1310-1317.
46. Chan YC, Siu CW, Lau YM, et al. Synergistic effects of inward rectifier (I) and pacemaker (I) currents on the induction of bioengineered cardiac automaticity.. J Cardiovasc Electrophysiol 2009; 20: 1048-1054.

47. Siu CW, et al. Hen-encoded pacemaker channels: From physiology and biophysics to bioengineering. J Membr Biol 2006; 214: 115-122.

48. Xue T, et al. Mechanistic role of $i(f)$ revealed by induction of ventricular automaticity by somatic gene transfer of gating-engineered pacemaker ( $\mathrm{h}$ (n) channels. Circulation 2007; 115: 1839-1850.

49. Wang K, et al. Electrophysiological properties of pluripotent human and mouse embryonic stem cells. Stem Cells 2005; 23: 1526-1534.

50. Jiang P, et al. Electrophysiological properties of human induced pluripotent stem cells. Am J Physiol Cell Physiol 2010; 298: C486-495.

51. Zhang J, et al. Functional cardiomyocytes derived from human induced pluripotent stem cells. Circ Res 2009; 104: e30-41.

52. Boyett MR, et al. The sinoatrial node, a heterogeneous pacemaker structure. Cardiovasc Res 2000; 47: 658-687.

53. Dobrzynski H, et al. New insights into pacemaker activity: Promoting understanding of sick sinus syndrome. Circulation 2007; 115: 1921-1932.

54. Dubin AM, Berul CI. Electrophysiological interventions for treatment of congestive heart failure in pediatrics and congenital heart disease. Expert Rev Cardiovasc Ther 2007; 5: 111-118.

55. Silka MJ, Bar-Cohen Y. Pacemakers and implantable cardioverter-defibrillators in pediatric patients. Heart Rhythm 2006; 3: 1360-1366.

56. Walsh EP, Cecchin F. Recent advances in pacemaker and implantable defibrillator therapy for young patients. Curr Opin Cardiol 2004; 19: 91-96.

57. Berul CI, Cecchin F. Indications and techniques of pediatric cardiac pacing. Expert Rev Cardiovasc Ther 2003; 1: 165-176.

58. Sliz NB, Jr., Johns JA. Cardiac pacing in infants and children. Cardiol Rev 2000; 8 : 223-239.

59. Miake J, et al. Gene therapy: Biological pacemaker created by gene transfer. Nature 2002; 419: 132-133.

60. Tse HF, et al. Bioartificial sinus node constructed via in vivo gene transfer of an engineered pacemaker hon channel reduces the dependence on electronic pacemaker in a sick-sinus syndrome model. Circulation 2006; 114: 1000-1011.

61. Robinson RB, et al. I(f) and the biological pacemaker. Pharmacol Res 2006; 53: 407-415.

62. Qu J, et al. Hcn2 overexpression in newborn and adult ventricular myocytes: Distinct effects on gating and excitability. Circ Res 2001; 89: E8-14.

63. Lesso H, Li RA. Helical secondary structure of the external s3-s4 linker of pacemaker (hcn) channels revealed by site-dependent perturbations of activation phenotype. J Biol Chem 2003; 278: 22290-22297.

64. Tsang SY, et al. Critical intra-linker interactions of hen1-encoded pacemaker channels revealed by interchange of s3-s4 determinants. Biochem Biophys Res Commun 2004; 322: 652-658.

65. Tsang SY, et al. Dissecting the structural and functional roles of the s3-s4 linker of pacemaker (hyperpolarization-activated cyclic nucleotide-modulated) channels by systematic length alterations. J Biol Chem 2004; 279: 43752-43759.

66. Kehat I, et al. Electromechanical integration of cardiomyocytes derived from human embryonic stem cells. Nat Biotechnol 2004; 22: 1282-1289.

67. Plotnikov AN, et al. Xenografted adult human mesenchymal stem cells provide a platform for sustained biological pacemaker function in canine heart. Circulation 2007; 116: 706-713.

68. Plotnikov AN, et al. Biological pacemaker implanted in canine left bundle branch provides ventricular escape rhythms that have physiologically acceptable rates. Circulation 2004; 109: 506-512.

69. Cho HC, et al. Creation of a biological pacemaker by cell fusion. Circulation Res 2007; 100: 1112-1115.

70. Alvarez-Dolado M, et al. Fusion of bone-marrow-derived cells with purkinje neurons, cardiomyocytes and hepatocytes. Nature 2003; 425: 968-973.

71. Gussoni E, et al. Long-term persistence of donor nuclei in a duchenne muscular dystrophy patient receiving bone marrow transplantation. J Clin Invest 2002; 110: 807-814.

72. Weimann JM, et al. Stable reprogrammed heterokaryons form spontaneously in purkinje neurons after bone marrow transplant. Nat Cell Biol 2003; 5: 959-966. 